DESY 14-007

ISSN 0418-9833

May 2014

\title{
Propagator mixing renormalization for Majorana fermions
}

\author{
Bernd A. Kniehl \\ II. Institut für Theoretische Physik, Universität Hamburg, \\ Luruper Chaussee 149, 22761 Hamburg, Germany
}

\begin{abstract}
We consider a mixed system of unstable Majorana fermions in a general paritynonconserving theory and renormalize its propagator matrix to all orders in the pole scheme, in which the squares of the renormalized masses are identified with the complex pole positions and the wave-function renormalization matrices are adjusted in compliance with the Lehmann-Symanzik-Zimmermann reduction formalism. In contrast to the case of unstable Dirac fermions, the wave-function renormalization matrices of the in and out states are uniquely fixed, while they again bifurcate in the sense that they are no longer related by pseudo-Hermitian conjugation. We present closed analytic expressions for the renormalization constants in terms of the scalar, pseudoscalar, vector, and pseudovector parts of the unrenormalized self-energy matrix, which is computable from the one-particle-irreducible Feynman diagrams of the flavor transitions, as well as their expansions through two loops. In the case of stable Majorana fermions, the well-known one-loop results are recovered.
\end{abstract}

PACS numbers: 11.10.Gh, 11.15.Bt, 12.15.Ff, 12.15.Lk 


\section{Introduction}

The Standard Model (SM) of elementary particle physics has been enormously consolidated by the discovery [1] at the CERN Large Hadron Collider of a new weak neutral resonance that, within the present experimental precision, shares the spin, parity, and charge-conjugation quantum numbers $J^{P C}=0^{++}$and the coupling strengths with the SM Higgs boson $H$, in the complete absence of any signals of new physics beyond the SM. Moreover, its mass of $(125.6 \pm 0.3) \mathrm{GeV}$ lies well inside the $M_{H}$ range predicted within the SM through global analyses of electroweak (EW) precision data, and it almost perfectly coincides with state-of-the-art determinations, based on three-loop evolution and two-loop matching, of the $M_{H}$ lower bound, $(129.6 \pm 1.5) \mathrm{GeV}[2,3$, from the requirement that the SM vacuum be stable way up to the scale of the Planck mass [4]. If the pole mass $m_{t}$ of the top quark, which, in want of a rigorous determination at the quantum level, is presently identified with a Monte-Carlo parameter [5], were just lower by an amount of the order of its total decay width $\Gamma_{t}=(2.0 \pm 0.5) \mathrm{GeV}$ [5], then the agreement would be perfect, implying that EW symmetry breaking is likely to be determined by Planck-scale physics [2]. In a way, this would solve the longstanding hierarchy problem of the SM. The Nobel Prize in Physics 2013 was recently awarded jointly to Englert and Higgs for the theoretical discovery of the Higgs mechanism.

Despite the recent triumph of the SM, we must keep in mind that its neutrino sector is still holding great longstanding mysteries. Among the most prominent ones of them is the question whether the neutrinos are Dirac or Majorana fermions [6]. In the latter case, physics beyond the SM is indispensable. On the other hand, numerous beyond-SM scenarios, in particular those in which the new physics is accessed via a Higgs portal, involve heavy Majorana neutrinos as ingredients to explain the smallness of the masses of the observed neutrinos via the seesaw mechanism [7]. In the ongoing endeavor to complete the all-order renormalization of the SM and its most favorable extensions among those not yet excluded experimentally, it is, therefore, necessary to also accommodate Majorana fermions allowing for flavor mixing and instability.

The renormalizability of the spontaneously broken quantum gauge theory underlying the SM was proven in 1971 [8], and the Nobel Prize in Physics 1999 was awarded to 't Hooft and Veltman for elucidating the quantum structure of $E W$ interactions in physics. The on-shell renormalization scheme, which includes the physical particle masses and Sommerfeld's fine-structure constant among the basic parameters, provides a natural framework for that. It was systematically elaborated at one loop for stable particles in Refs. [9, 10, 11, 12], and a particularly useful variant of it was proposed in Ref. [13]. The on-shell renormalization of the SM was established to all orders of perturbation theory using the algebraic method [14. However, all particles were assumed to be stable, neutrinos were taken to be massless, and quark flavor mixing was neglected. To eliminate these unrealistic assumptions, one needs to develop a pole scheme of mixing renormalization for unstable particles valid to all orders. Apart from being conceptually desirable, this is becoming of major phenomenological importance, both in the SM and beyond, even more so because mixing and instability of elementary particles prevail and concur 
in nature. This requires generalized concepts for flavor-changing propagators and vertices. In the SM with massless neutrinos, these are the propagator matrices of the upand down-type quarks and their charged-current vertices, which involve the CabibboKobayashi-Maskawa (CKM) [15] quark mixing matrix. This pattern carries over to the lepton sector if the neutrinos are massive Dirac fermions, and the analog of the CKM matrix is the Pontecorvo-Maki-Nakagawa-Sakata [16] neutrino mixing matrix. Things are more complicated in the presence of Majorana degrees of freedom in the neutrino sector, which typically give rise to flavor-changing vertices involving the $Z^{0}$ and Higgs bosons, too.

As for the renormalization of propagator matrices of mixed systems of fermions, the situation is as follows. In Ref. [17], an early treatment of finite renormalization effects both for quarks in hadronic bound states and leptons may be found. In Ref. [18], the ultraviolet (UV) renormalization of the fermion masses was considered, and the pole masses were shown to be gauge independent to all orders in the SM using Nielsen identities [19], both for stable and unstable Dirac fermions. In Ref. [20], the UV renormalization of the Dirac fermion fields was discussed for the case of stability, and the dressed propagator matrices were written in closed form, both for the unrenormalized and renormalized versions. Furthermore, it was explicitly proven that the wave-function renormalization (WFR) conditions proposed by Aoki, Hioki, Kawabe, Konuma, and Muta (AHKKM) [10] guarantee the unit-residue properties of the diagonal elements of the renormalized propagator matrix to all orders, in compliance with the Lehmann-Symnanzik-Zimmermann (LSZ) reduction formalism [21]. In Refs. [22,23], the discussion of Ref. [20] was extended to the case of unstable Dirac fermions, and closed all-order expressions for their mass conterterms and WFR matrices were constructed. The purpose of the present paper is to generalize the approach of Refs. [22,23] to Majorana fermions. Specifically, we work out the renormalization of the propagator matrix of a mixed system of unstable Majorana fermions to all orders.

As for the flavor mixing matrices of Majorana fermions, various renormalization prescriptions have been proposed at one loop for the case of stability [24,25,26]. Specifically, the approach of Ref. [26] is based on Ref. [27]. As pointed out in Ref. [25], necessary conditions for the renormalized fermion mixing matrices include UV finiteness, gauge independence, and (pseudo)unitarity. Furthermore, it is desirable for their counterterms to be on shell, flavor democratic, finite in the case of fermion mass degeneracy, and expressible in terms of self-energies only [28].

This paper is organized as follows. In Sec. 2, we start from the inverse of the unrenormalized propagator matrix and obtain the dressed propagator matrix by performing the Dyson resummation [29]. At this point, we define the renormalization conditions for the complex pole masses in terms of secular equations and solve them to all orders of perturbation theory. In Sec. 3, we introduce the WFR matrices, explain how they enter the dressed propagator matrix, and define renormalized self-energies in such a way that the renormalized propagator matrix emerges from its unrenormalized counterpart by replacing the unrenormalized self-energies in the latter by their renormalized counterparts. In Sec. 4, we generalize the AHKKM WFR conditions [10] to the case of instability, impose 
them on the inverse of the renormalized propagator matrix obtained in Sec. 3, and solve them exactly for the WFR matrices, so as to establish them in closed analytic form valid to all orders of perturbation theory. In contrast to the Dirac case [22,23], the WFR matrices are uniquely determined. A similar observation was made for the case of stability at one loop 24. The generalized AHKKM renormalization conditions also allow us to find an alternative all-order expression for the pole mass counterterms. In Sec. 5, we demonstrate that WFR bifurcation is an inevitable consequence of the LSZ condition [21] for unstable Majorana fermions. Similar observations were made for unstable Dirac fermions at the one-loop order [30] and to all orders [22,23]. In Sec. 6, we expand the all-order expressions for the renormalization constants derived in Secs. 24 through two loops and cast them into a form ready to use in phenomenological calculations. As a by-product, we recover the one-loop results for the case of stability [24]. Section 7 contains a summary and an outlook.

\section{Unrenormalized dressed propagator matrix}

We consider a system of $N$ unstable Majorana fermions in the context of some general parity-nonconserving theory with intergeneration mixing. We denote the bare quantum fields of their flavor eigenstates by $\psi_{i}^{\prime 0}(x)$, where the subscript $i=1, \ldots, N$ is the flavor index and the superscript 0 labels bare quantities. For the sake of a compact notation, we group them into a column vector in flavor space,

$$
\Psi^{\prime 0}(x)=\left(\begin{array}{c}
\psi_{1}^{\prime 0}(x) \\
\vdots \\
\psi_{N}^{\prime 0}(x)
\end{array}\right) .
$$

The Majorana nature of a fermion to be at the same time its own antiparticle manifests itself in the condition 1

$$
\Psi^{\prime 0}(x)=C\left[\bar{\Psi}^{\prime 0}(x)\right]^{T},
$$

where $C$ is a unitary matrix in four-dimensional spinor space, with $C^{-1}=C^{\dagger}$, which transforms Dirac's $\gamma^{\mu}$ matrices as $C \gamma^{\mu T} C^{\dagger}=-\gamma^{\mu}$. From the definition of the $\gamma_{5}$ matrix, $\gamma_{5}=i \gamma^{0} \gamma^{1} \gamma^{2} \gamma^{3}$, it follows that $C \gamma_{5}^{T} C^{\dagger}=\gamma_{5}$. Because $\bar{\Psi}^{\prime 0}(x)=\left[\Psi^{\prime 0}(x)\right]^{\dagger} \gamma^{0}$ may also be evaluated from Eq. (2) as $\bar{\Psi}^{\prime 0}(x)=\left[\Psi^{\prime 0}(x)\right]^{T} C^{*}, C$ must satisfy the additional condition $C^{\dagger}=-C^{*}$, by being antisymmetric, $C^{T}=-C$. The kinetic term of the bare Lagrangian is

$$
\mathcal{L}^{0}(x)=\frac{1}{2}\left[\Psi^{\prime 0}(x)\right]^{T} C^{*}\left(i \not \partial-\mathcal{M}^{\prime 0}\right) \Psi^{\prime 0}(x),
$$

where $\mathcal{M}^{\prime 0}$ is the bare mass matrix and the overall factor $1 / 2$ corrects for the seeming paradox that each Majorana fermion contributes to the total energy twice, as a particle and an antiparticle. For $\mathcal{L}^{0}(x)$ to be Hermitian, $\left[\mathcal{L}^{0}(x)\right]^{*}=\mathcal{L}^{0}(x), \mathcal{M}^{\prime 0}$ must satisfy

\footnotetext{
${ }^{1}$ Here and in the following, the superscript $T$ implies simultaneous transposition in the spinor and generation spaces.
} 
the pseudo-Hermiticity relation $\gamma^{0} \mathcal{M}^{\prime 0 \dagger} \gamma^{0}=\mathcal{M}^{\prime 0}$, which constrains it to the form $\mathcal{M}^{\prime 0}=$ $M^{\prime 0} a_{+}+M^{\prime 0 \dagger} a_{-}$, where $M^{\prime 0}$ is an arbitrary complex $N \times N$ matrix and $a_{ \pm}=\left(I_{4} \pm \gamma_{5}\right) / 2$ are the chiral projection operators. Here and in the following, $I_{n}$ denotes the $n \times n$ unit matrix. Exploiting the identity $\left[\mathcal{L}^{0}(x)\right]^{T}=\mathcal{L}^{0}(x)$ in connection with the Grassmannian property of fermionic quantum fields, we obtain the additional relation $C \mathcal{M}^{\prime 0 T} C^{\dagger}=\mathcal{M}^{\prime 0}$, which implies that $M^{\prime 0}$ is symmetric, $M^{\prime 0 T}=M^{\prime 0}$. By Autonne-Takagi matrix factorization, 2 the complex, symmetric $N \times N$ matrix $M^{\prime 0}$ may be transformed into a real, diagonal matrix $M^{0}$ with nonnegative entries 3

$$
M_{i j}^{0}=m_{i}^{0} \delta_{i j}
$$

via a single unitary matrix $U_{-}$, as $M^{0}=U_{-} M^{\prime 0} U_{-}^{T}$. The bare field multiplet of the mass eigenstates $\psi_{i}^{0}(x)$, with bare masses $m_{i}^{0}$, is then given by $\Psi^{0}(x)=U \Psi^{\prime 0}(x)$, where $U=U_{-}^{*} a_{+}+U_{-} a_{-}$. Upon this field transformation, Eq. (3) indeed assumes the standard form

$$
\begin{aligned}
\mathcal{L}^{0}(x)= & \frac{1}{2}\left[\Psi^{0}(x)\right]^{T} C^{*}\left(i \not \partial-M^{0}\right) \Psi^{0}(x) \\
= & \frac{1}{2}\left\{i\left[\Psi_{-}^{0}(x)\right]^{T} C^{*} \not \partial \Psi_{+}^{0}(x)+i\left[\Psi_{+}^{0}(x)\right]^{T} C^{*} \not \partial \Psi_{-}^{0}(x)\right. \\
& \left.-\left[\Psi_{+}^{0}(x)\right]^{T} C^{*} M^{0} \Psi_{+}^{0}(x)-\left[\Psi_{-}^{0}(x)\right]^{T} C^{*} M^{0} \Psi_{-}^{0}(x)\right\},
\end{aligned}
$$

where $\Psi_{ \pm}^{0}(x)=a_{ \pm} \Psi^{0}(x)$ are the right- and left-handed field components, respectively. Owing to the identity $\gamma^{0} C U^{*} C^{\dagger} \gamma^{0}=U$, the Majorana property of the weak eigenstates in Eq. (2) carries over to the mass eigenstates,

$$
\Psi^{0}(x)=C\left[\bar{\Psi}^{0}(x)\right]^{T} .
$$

However, it does not hold separately for $\Psi_{ \pm}^{0}(x)$. Instead, we have $\Psi_{ \pm}^{0}(x)=C\left[\bar{\Psi}_{\mp}^{0}(x)\right]^{T}$ [24].

In momentum space, the unrenormalized propagator matrix is defined as

$$
i P(\not p)=\int d^{4} x e^{i p \cdot x}\left\langle 0\left|T\left\{\Psi^{0}(x) \otimes\left[\Psi^{0}(0)\right]^{T} C^{*}\right\}\right| 0\right\rangle,
$$

where $T$ is the time-ordered product and $\otimes$ is to indicate a tensorial product both in the spinor and generation spaces. Its inverse is built up by the one-particle-irreducible Feynman diagrams contributing to the transitions $j \rightarrow i$ and has the form

$$
[P(\not p)]^{-1}=\not p-M^{0}-\Sigma(\not p),
$$

where $\Sigma(\not p)$ is the unrenormalized self-energy matrix. By Lorentz covariance, the latter exhibits the structure

$$
\Sigma(\not p)=\left[A_{+}\left(p^{2}\right)+\not p B_{+}\left(p^{2}\right)\right] a_{+}+(+\leftrightarrow-),
$$

\footnotetext{
${ }^{2}$ An explicit proof of this theorem may be found, e.g., in Appendix B of Ref. [26].

${ }^{3}$ In this paper, summation over repeated indices is not implied in the absence of summation symbols.
} 
where the entries in the matrices $A_{ \pm}\left(p^{2}\right)$ and $B_{ \pm}\left(p^{2}\right)$ are Lorentz-invariant functions of $p^{2}$. The latter may be calculated from the bare Lagrangian order by order in perturbation theory. However, we refrain from explicitly performing perturbative expansions in the following, rendering our results valid to all orders. Defining

$$
S_{ \pm}\left(p^{2}\right)=I_{N}-B_{ \pm}\left(p^{2}\right), \quad T_{ \pm}\left(p^{2}\right)=M^{0}+A_{ \pm}\left(p^{2}\right),
$$

Eq. (8) may be cast into a compact form,

$$
[P(\not p)]^{-1}=\left[\not p S_{+}\left(p^{2}\right)-T_{+}\left(p^{2}\right)\right] a_{+}+(+\leftrightarrow-) .
$$

The one-particle-reducible Feynman diagrams may be collected systematically by performing the Dyson resummation [29], which is equivalent to inverting Eq. (11) and yields [20]

$$
\begin{aligned}
P(\not p) & =\left[\not p+D_{-}\left(p^{2}\right)\right] S_{-}^{-1}\left(p^{2}\right)\left[p^{2}-E_{-}\left(p^{2}\right)\right]^{-1} a_{+}+(+\leftrightarrow-) \\
& =a_{+}\left[p^{2}-F_{+}\left(p^{2}\right)\right]^{-1} S_{+}^{-1}\left(p^{2}\right)\left[\not p+C_{+}\left(p^{2}\right)\right]+(+\leftrightarrow-),
\end{aligned}
$$

with the short-hand notations

$$
\begin{array}{lll}
C_{ \pm}\left(p^{2}\right)=T_{\mp}\left(p^{2}\right) S_{\mp}^{-1}\left(p^{2}\right), & & D_{ \pm}\left(p^{2}\right)=S_{\mp}^{-1}\left(p^{2}\right) T_{ \pm}\left(p^{2}\right), \\
E_{ \pm}\left(p^{2}\right)=C_{ \pm}\left(p^{2}\right) C_{\mp}\left(p^{2}\right), & & F_{ \pm}\left(p^{2}\right)=D_{\mp}\left(p^{2}\right) D_{ \pm}\left(p^{2}\right) .
\end{array}
$$

In fact, Eqs. (11) and (12) are easily seen to satisfy $P(\not p)[P(\not p)]^{-1}=[P(\not p)]^{-1} P(\not p)=I_{N} \otimes I_{4}$. From the first equality in Eq. (10) it follows that $\operatorname{det}\left[S_{ \pm}\left(p^{2}\right)\right]=1+\mathcal{O}(\alpha) \neq 0$ with $\alpha$ being a generic coupling constant, so that we may evaluate $S_{ \pm}^{-1}\left(p^{2}\right)$ as $S_{ \pm}^{-1}\left(p^{2}\right)=$ $\operatorname{adj}\left[S_{ \pm}\left(p^{2}\right)\right] / \operatorname{det}\left[S_{ \pm}\left(p^{2}\right)\right]$ A Alternatively, we may compute $S_{ \pm}^{-1}\left(p^{2}\right)$ perturbatively as a geometric series, $S_{ \pm}^{-1}\left(p^{2}\right)=I_{N}+\sum_{n=1}^{\infty} B_{ \pm}^{n}\left(p^{2}\right)$.

Since the four matrices $\left[p^{2}-E_{ \pm}\left(p^{2}\right)\right]$ and $\left[p^{2}-F_{ \pm}\left(p^{2}\right)\right]$, the inverses of which appear in the individual propagator parts in Eq. (12), are related by similarity transformations,

$$
\begin{aligned}
p^{2}-E_{+}\left(p^{2}\right) & =C_{-}\left(p^{2}\right)\left[p^{2}-E_{-}\left(p^{2}\right)\right] C_{-}^{-1}\left(p^{2}\right) \\
& =S_{+}\left(p^{2}\right)\left[p^{2}-F_{+}\left(p^{2}\right)\right] S_{+}^{-1}\left(p^{2}\right) \\
& =T_{-}\left(p^{2}\right)\left[p^{2}-F_{-}\left(p^{2}\right)\right] T_{-}^{-1}\left(p^{2}\right),
\end{aligned}
$$

we have

$$
\operatorname{det}\left[p^{2}-E_{+}\left(p^{2}\right)\right]=\operatorname{det}\left[p^{2}-E_{-}\left(p^{2}\right)\right]=\operatorname{det}\left[p^{2}-F_{+}\left(p^{2}\right)\right]=\operatorname{det}\left[p^{2}-F_{-}\left(p^{2}\right)\right],
$$

\footnotetext{
${ }^{4}$ The adjugate (classical adjoint) $\operatorname{adj} A=C^{T}$ of a quadratic $(n \times n)$ matrix $A$ is the transpose of the matrix $C$ the elements $C_{i j}$ of which are the cofactors of the elements $A_{i j}$ of $A$. The cofactor $C_{i j}$ of the element $A_{i j}$ of $A$ is $(-1)^{i+j}$ times the determinant of the $(n-1) \times(n-1)$ matrix obtained by deleting the $i$ th row and the $j$ th column of $A$. The theorem $A \operatorname{adj} A=(\operatorname{adj} A) A=(\operatorname{det} A) I_{n}$ may be understood by observing that, according to Laplace's expansion formula, $\sum_{k=1}^{n} A_{i k} C_{j k}$ is the determinant of the matrix obtained from $A$ by replacing the $j$ th row by the $i$ th row and $\sum_{k=1}^{n} C_{k i} A_{k j}$ is the determinant of the matrix obtained from $A$ by replacing the $i$ th column by the $j$ th column. If $i=j$, then, in both cases, the result is just $\operatorname{det} A$. If $i \neq j$, then it is zero because these determinants have two identical rows and columns, respectively. If $\operatorname{det} A \neq 0$, then $A^{-1}=(\operatorname{det} A)^{-1}$ adj $A$.
} 
which, by virtue of footnote 4, guarantees that the individual propagator parts all have poles at the same (complex) positions $p^{2}=M_{i}^{2}$ defined as the zeros of Eq. (15) by any of the secular equations [17,18,20]

$$
\operatorname{det}\left[M_{i}^{2}-E_{ \pm}\left(M_{i}^{2}\right)\right]=\operatorname{det}\left[M_{i}^{2}-F_{ \pm}\left(M_{i}^{2}\right)\right]=0 .
$$

Here, $M_{i}$ is the complex pole mass of Majorana fermion $i$. It is related to the real pole mass $m_{i}$ and total decay width $\Gamma_{i}$ as 31,32

$$
M_{i}=m_{i}-i \frac{\Gamma_{i}}{2}
$$

In the Appendix of Ref. [20], Eq. (16) is solved perturbatively through two loops for the case of stable Dirac fermions. Here, we derive closed all-order expressions for $M_{i}$ in terms of the Lorentz-invariant functions $\left[A_{ \pm}\left(p^{2}\right)\right]_{i j}$ and $\left[B_{ \pm}\left(p^{2}\right)\right]_{i j}$. Owing to footnote 4 , we have the identities

$$
\begin{aligned}
& \left\{\left[p^{2}-F_{ \pm}\left(p^{2}\right)\right] \operatorname{adj}\left[p^{2}-F_{ \pm}\left(p^{2}\right)\right]\right\}_{i i}=\operatorname{det}\left[p^{2}-F_{ \pm}\left(p^{2}\right)\right], \\
& \left\{\operatorname{adj}\left[p^{2}-E_{ \pm}\left(p^{2}\right)\right]\left[p^{2}-E_{ \pm}\left(p^{2}\right)\right]\right\}_{i i}=\operatorname{det}\left[p^{2}-E_{ \pm}\left(p^{2}\right)\right] .
\end{aligned}
$$

At this point, we introduce the two matrices,

$$
M_{i j}^{ \pm}=\left\{\operatorname{adj}\left[M_{j}^{2}-F_{ \pm}\left(M_{j}^{2}\right)\right]\right\}_{i j}, \quad \bar{M}_{i j}^{ \pm}=\left\{\operatorname{adj}\left[M_{i}^{2}-E_{ \pm}\left(M_{i}^{2}\right)\right]\right\}_{i j},
$$

which we shall need again later, and observe that $M_{i i}^{ \pm}, \bar{M}_{i i}^{ \pm}=\prod_{j \neq i}\left(M_{i}^{2}-M_{j}^{2}\right)+\mathcal{O}(\alpha) \neq 0$. For the solution $p^{2}=M_{i}^{2}$ of Eq. (16), we then obtain from Eq. (18) the exact expression

$$
M_{i}^{2}=\frac{\left[F_{ \pm}\left(M_{i}^{2}\right) M^{ \pm}\right]_{i i}}{M_{i i}^{ \pm}}=\frac{\left[\bar{M}^{ \pm} E_{ \pm}\left(M_{i}^{2}\right)\right]_{i i}}{\bar{M}_{i i}^{ \pm}} .
$$

We shall see later that the WFR procedure generates yet another closed all-order expression for $M_{i}$, namely, the one specified in Eq. (51), in which both matrices of Eq. (19) enter in a symmetric way.

Equations (8)-(20) also apply to unstable Dirac fermions as they stand and were partly presented in Refs. 2223]. However, there are additional constraints for Majorana fermions. In fact, making use of the Grassmannian nature of fermionic quantum fields in Eq. (7), we find

$$
C[P(-\not p)]^{T} C^{\dagger}=P(\not p) .
$$

Applying Eq. (21) to Eq. (8), we obtain $C[\Sigma(-\not p)]^{T} C^{\dagger}=\Sigma(\not p)$, which implies via Eqs. (9), (10), (13), and (19) that

$$
\begin{array}{llll}
A_{ \pm}^{T}\left(p^{2}\right)=A_{ \pm}\left(p^{2}\right), & B_{ \pm}^{T}\left(p^{2}\right)=B_{\mp}\left(p^{2}\right), & S_{ \pm}^{T}\left(p^{2}\right)=S_{\mp}\left(p^{2}\right), & T_{ \pm}^{T}\left(p^{2}\right)=T_{ \pm}\left(p^{2}\right), \\
C_{ \pm}^{T}\left(p^{2}\right)=D_{\mp}\left(p^{2}\right), & E_{ \pm}^{T}\left(p^{2}\right)=F_{\mp}\left(p^{2}\right), & \left(M^{ \pm}\right)^{T}=\bar{M}^{\mp}, &
\end{array}
$$

where we have used $\operatorname{adj}\left(A^{T}\right)=(\operatorname{adj} A)^{T}$, which follows from footnote 4 , in the last equality. The first equality in Eq. (22) agrees with the second equality in Eq. (4.7) of Ref. [24]. It is interesting to observe that the last equality in Eq. (20) may be derived from the last two equalities in Eq. (22) alone, without recourse to Eq. (18), by noticing that diagonal matrix elements are invariant under transposition. 


\section{Renormalized dressed propagator matrix}

In the following, we adopt the pole renormalization scheme, in which the complex pole masses $M_{i}$ serve as the renormalized masses; i.e., the mass counterterms $\delta M_{i}$ are fixed by the relations

$$
m_{i}^{0}=M_{i}+\delta M_{i}
$$

The field renormalization is implemented by writing

$$
\Psi^{0}(x)=Z^{1 / 2} \Psi(x)
$$

where $\Psi(x)$ is the renormalized field multiplet and

$$
Z^{1 / 2}=Z_{+}^{1 / 2} a_{+}+Z_{-}^{1 / 2} a_{-},
$$

with $Z_{ \pm}^{1 / 2}$ being the WFR matrices. From Eq. (24), it follows that

$$
\left[\Psi^{0}(x)\right]^{T} C^{*}=[\Psi(x)]^{T} C^{*} \bar{Z}^{1 / 2}
$$

where

$$
\bar{Z}^{1 / 2}=C Z^{T 1 / 2} C^{\dagger}=a_{+} Z_{+}^{T 1 / 2}+a_{-} Z_{-}^{T 1 / 2} .
$$

Solving Eqs. (24) and (26) for the renormalized field multiplets, we have

$$
\Psi(x)=Z^{-1 / 2} \Psi^{0}(x), \quad[\Psi(x)]^{T} C^{*}=\left[\Psi^{0}(x)\right]^{T} C^{*} \bar{Z}^{-1 / 2},
$$

where

$$
Z^{-1 / 2}=Z_{+}^{-1 / 2} a_{+}+Z_{-}^{-1 / 2} a_{-}, \quad \bar{Z}^{-1 / 2}=a_{+} Z_{+}^{T-1 / 2}+a_{-} Z_{-}^{T-1 / 2}
$$

are the inverses of the matrices in Eqs. (25) and (27), respectively. Using Eq. (28), we may express the renormalized propagator matrix,

$$
i \hat{P}(\not p)=\int d^{4} x e^{i p \cdot x}\left\langle 0\left|T\left\{\Psi(x) \otimes[\Psi(0)]^{T} C^{*}\right\}\right| 0\right\rangle,
$$

in terms of the unrenormalized one in Eq. (17) as

$$
\hat{P}(\not p)=Z^{-1 / 2} P(\not p) \bar{Z}^{-1 / 2}
$$

Substituting Eq. (12) in Eq. (31), we thus obtain

$$
\begin{aligned}
\hat{P}(\not p) & =\left[Z_{-}^{-1 / 2} \not p+Z_{+}^{-1 / 2} D_{-}\left(p^{2}\right)\right] S_{-}^{-1}\left(p^{2}\right)\left[p^{2}-E_{-}\left(p^{2}\right)\right]^{-1} Z_{+}^{T-1 / 2} a_{+}+(+\leftrightarrow-) \\
& =a_{+} Z_{+}^{-1 / 2}\left[p^{2}-F_{+}\left(p^{2}\right)\right]^{-1} S_{+}^{-1}\left(p^{2}\right)\left[\not p Z_{-}^{T-1 / 2}+C_{+}\left(p^{2}\right) Z_{+}^{T-1 / 2}\right]+(+\leftrightarrow-) .
\end{aligned}
$$

We may absorb the WFR matrices in Eq. (32) by defining renormalized counterparts of $S_{ \pm}\left(p^{2}\right)$ and $T_{ \pm}\left(p^{2}\right)$ in Eq. (10) as

$$
\hat{S}_{ \pm}\left(p^{2}\right)=Z_{\mp}^{T^{1 / 2}} S_{ \pm}\left(p^{2}\right) Z_{ \pm}^{1 / 2}, \quad \hat{T}_{ \pm}\left(p^{2}\right)=Z_{ \pm}^{T 1 / 2} T_{ \pm}\left(p^{2}\right) Z_{ \pm}^{1 / 2}
$$


In analogy to Eq. (13), we are thus led to define

$$
\begin{aligned}
& \hat{C}_{ \pm}\left(p^{2}\right)=\hat{T}_{\mp}\left(p^{2}\right) \hat{S}_{\mp}^{-1}\left(p^{2}\right)=Z_{\mp}^{T 1 / 2} C_{ \pm}\left(p^{2}\right) Z_{ \pm}^{T-1 / 2} \\
& \hat{D}_{ \pm}\left(p^{2}\right)=\hat{S}_{\mp}^{-1}\left(p^{2}\right) \hat{T}_{ \pm}\left(p^{2}\right)=Z_{\mp}^{-1 / 2} D_{ \pm}\left(p^{2}\right) Z_{ \pm}^{1 / 2} \\
& \hat{E}_{ \pm}\left(p^{2}\right)=\hat{C}_{ \pm}\left(p^{2}\right) \hat{C}_{\mp}\left(p^{2}\right)=Z_{\mp}^{T 1 / 2} E_{ \pm}\left(p^{2}\right) Z_{\mp}^{T-1 / 2} \\
& \hat{F}_{ \pm}\left(p^{2}\right)=\hat{D}_{\mp}\left(p^{2}\right) \hat{D}_{ \pm}\left(p^{2}\right)=Z_{ \pm}^{-1 / 2} F_{ \pm}\left(p^{2}\right) Z_{ \pm}^{1 / 2}
\end{aligned}
$$

Thus, Eq. (32) becomes

$$
\begin{aligned}
\hat{P}(\not p) & =\left[\not p+\hat{D}_{-}\left(p^{2}\right)\right] \hat{S}_{-}^{-1}\left(p^{2}\right)\left[p^{2}-\hat{E}_{-}\left(p^{2}\right)\right]^{-1} a_{+}+(+\leftrightarrow-) \\
& =a_{+}\left[p^{2}-\hat{F}_{+}\left(p^{2}\right)\right]^{-1} \hat{S}_{+}^{-1}\left(p^{2}\right)\left[\not p+\hat{C}_{+}\left(p^{2}\right)\right]+(+\leftrightarrow-) .
\end{aligned}
$$

By observing from the last two lines of Eq. (34) that

$$
\operatorname{det}\left[p^{2}-\hat{E}_{ \pm}\left(p^{2}\right)\right]=\operatorname{det}\left[p^{2}-E_{ \pm}\left(p^{2}\right)\right], \quad \operatorname{det}\left[p^{2}-\hat{F}_{ \pm}\left(p^{2}\right)\right]=\operatorname{det}\left[p^{2}-F_{ \pm}\left(p^{2}\right)\right],
$$

we understand that the pole positions $M_{i}^{2}$ are not affected by the WFR, as it should be [18. Mutatis mutandis, the inverse of the renormalized propagator matrix reads

$$
\begin{aligned}
{[\hat{P}(\not p)]^{-1} } & =\left[\not p \hat{S}_{+}\left(p^{2}\right)-\hat{T}_{+}\left(p^{2}\right)\right] a_{+}+(+\leftrightarrow-) \\
& =\left[Z_{-}^{T 1 / 2} \not p S_{+}\left(p^{2}\right)-Z_{+}^{T 1 / 2} T_{+}\left(p^{2}\right)\right] Z_{+}^{1 / 2} a_{+}+(+\leftrightarrow-) .
\end{aligned}
$$

The counterparts of Eqs. (26) and (27) for unstable Dirac fermions read [22,23]

$$
\bar{\Psi}^{0}(x)=\bar{\Psi}(x) \bar{Z}^{1 / 2}
$$

and

$$
\bar{Z}^{1 / 2}=a_{-} \bar{Z}_{+}^{1 / 2}+a_{+} \bar{Z}_{-}^{1 / 2},
$$

while the relationship in the first equality of Eq. (27) does not hold then. Nevertheless, Eqs. (32)-(35) and (37) may be recovered from Ref. [23] via the substitution

$$
\bar{Z}_{ \pm}^{1 / 2}=Z_{\mp}^{T 1 / 2}
$$

which may be gleaned by comparing Eqs. (27) and (39).

\section{Generalized WFR conditions}

Similarly to the case of unstable Dirac fermions [22]23], we determine the WFR matrices $Z_{ \pm}^{1 / 2}$ by requiring that, when any Majorana fermion $n$ approaches its mass shell, $\not p \rightarrow M_{n}$, the respective diagonal element $[\hat{P}(\not p)]_{n n}$ of the renormalized propagator resonates with unit residue, while all the other elements stay finite, i.e.,

$$
[\hat{P}(\not p)]_{i j}=\frac{\delta_{i n} \delta_{n j}}{\not p-M_{n}}+\mathcal{O}(1)
$$


in accordance with the LSZ reduction formalism [21]. The behavior of $\hat{P}(\not p)$ in Eq. (41) necessitates that $[\hat{P}(\not p)]^{-1}$ behaves as

$$
\left\{[\hat{P}(\not p)]^{-1}\right\}_{i j}=\left\{\begin{array}{lll}
\left(\not p-M_{n}\right)\left[I_{4}+\mathcal{O}\left(\not p-M_{n}\right)\right] & \text { if } \quad i=n=j, \\
{\left[M_{i n}+\mathcal{O}\left(\not p-M_{n}\right)\right]\left(\not p-M_{n}\right)} & \text { if } \quad i \neq n=j, \\
\left(\not p-M_{n}\right)\left[M_{n j}+\mathcal{O}\left(\not p-M_{n}\right)\right] & \text { if } \quad i=n \neq j, \\
M_{i j}+\mathcal{O}\left(\not p-M_{n}\right) & \text { if } i \neq n \neq j,
\end{array}\right.
$$

where $M_{i j}$ are constant matrices in spinor space, which, in general, do not commute with $\not p$. In fact, they are linear combinations of the Dirac matrices $I_{4}$ and $\gamma_{5}$ with constant coefficients. The specific structure of Eq. (42) may be easily understood by multiplying Eqs. (41) and (42) in both orders. The behavior in Eq. (42) may be arranged for by imposing the generalized version [22,23] of the on-shell WFR conditions [10],

$$
\begin{gathered}
\left\{[\hat{P}(\not p)]^{-1}\right\}_{i j} u\left(\vec{p}, M_{j}\right)=0, \\
\bar{u}\left(\vec{p}, M_{i}\right)\left\{[\hat{P}(\not p)]^{-1}\right\}_{i j}=0, \\
\left\{\frac{1}{\not p-M_{i}}\left\{[\hat{P}(\not p)]^{-1}\right\}_{i i}\right\} u\left(\vec{p}, M_{i}\right)=u\left(\vec{p}, M_{i}\right), \\
\bar{u}\left(\vec{p}, M_{i}\right)\left\{\left\{[\hat{P}(\not p)]^{-1}\right\}_{i i} \frac{1}{\not p-M_{i}}\right\}=\bar{u}\left(\vec{p}, M_{i}\right),
\end{gathered}
$$

for $i, j=1, \ldots, N$. Here, $u\left(\vec{p}, M_{i}\right)$ and $\bar{u}\left(\vec{p}, M_{i}\right)$ are four-component spinors satisfying the Dirac equations,

$$
\left(\not p-M_{i}\right) u\left(\vec{p}, M_{i}\right)=\bar{u}\left(\vec{p}, M_{i}\right)\left(\not p-M_{i}\right)=0 \text {. }
$$

For stable Dirac fermions, an explicit proof that Eq. (43) entails Eq. (41) may be found in Sec. III of Ref. [20]. This proof carries over to unstable Dirac fermions, as explained in Sec. VII of Ref. [23], and also to unstable Majorana fermions.

The WFR matrices $Z_{ \pm}^{1 / 2}$ may be determined by inserting Eq. (37) into Eq. (43) and proceeding along the lines of Refs. [22,23]. The results may be inferred from Refs. [22,23] via the substitution in Eq. (401). Using also the last equality in Eq. (22), we may translate Eqs. (57), (58), (71), and (72) of Ref. [23] as

$$
\begin{aligned}
\left(Z_{\mp}^{1 / 2}\right)_{i i}\left(Z_{ \pm}^{1 / 2}\right)_{i i} & =\frac{M_{i i}^{\mp} M_{i i}^{ \pm}}{s_{i}^{ \pm}\left(M_{i}^{2}\right)\left[1-f_{i}^{\prime}\left(M_{i}^{2}\right)\right]}, \\
\left(Z_{ \pm}^{1 / 2}\right)_{i i}^{2} & =\frac{M_{i}\left(M_{i i}^{ \pm}\right)^{2}}{t_{i}^{ \pm}\left(M_{i}^{2}\right)\left[1-f_{i}^{\prime}\left(M_{i}^{2}\right)\right]} \\
\left(Z_{ \pm}^{1 / 2}\right)_{i j} & =\frac{M_{i j}^{ \pm}}{M_{j j}^{ \pm}}\left(Z_{ \pm}^{1 / 2}\right)_{j j}
\end{aligned}
$$

where

$$
\begin{aligned}
s_{i}^{ \pm}\left(p^{2}\right) & =\left[\bar{M}^{ \pm} S_{ \pm}\left(p^{2}\right) M^{ \pm}\right]_{i i}, \\
t_{i}^{ \pm}\left(p^{2}\right) & =\left[\bar{M}^{\mp} T_{ \pm}\left(p^{2}\right) M^{ \pm}\right]_{i i}, \\
f_{i}\left(p^{2}\right) & =\frac{t_{i}^{+}\left(p^{2}\right) t_{i}^{-}\left(p^{2}\right)}{s_{i}^{+}\left(p^{2}\right) s_{i}^{-}\left(p^{2}\right)},
\end{aligned}
$$


and $M_{i j}^{ \pm}$and $\bar{M}_{i j}^{ \pm}$are defined in Eq. (19). Furthermore, Eq. (65) of Ref. [23] carries over as is,

$$
M_{i}^{2}=f_{i}\left(M_{i}^{2}\right) .
$$

As anticipated in the context of Eq. (20), Eq. (51) provides an alternative all-order expression for $M_{i}$. With the aid of the third and last equalities in Eq. (22), we observe that the two expressions in Eq. (48) actually coincide, so that we may omit the superscript \pm ,

$$
s_{i}\left(p^{2}\right)=s_{i}^{ \pm}\left(p^{2}\right)
$$

and rewrite Eq. (50) as

$$
f_{i}\left(p^{2}\right)=\frac{t_{i}^{+}\left(p^{2}\right) t_{i}^{-}\left(p^{2}\right)}{\left[s_{i}\left(p^{2}\right)\right]^{2}} .
$$

Furthermore, we notice that Eqs. (45) and (46) are redundant. In fact, Eq. (45) follows from Eq. (46) with the help of Eqs. (50) and (51). We conclude that, owing to the Majorana-induced constraint in Eq. (27), Eqs. (45)-(47) uniquely determine the WFR matrices to be

$$
\left(Z_{ \pm}^{1 / 2}\right)_{i j}=M_{i j}^{ \pm}\left(\frac{M_{j}}{t_{j}^{ \pm}\left(M_{j}^{2}\right)\left[1-f_{j}^{\prime}\left(M_{j}^{2}\right)\right]}\right)^{1 / 2} .
$$

In contrast, the renormalization conditions in Eq. (43) leave some residual freedom in the determination of the WFR matrices for unstable Dirac fermions, as explained in Refs. [22 23].

From Eqs. (23) and (151), we obtain the all-order mass counterterm as

$$
\delta M_{i}=m_{i}^{0}-\sqrt{f_{i}\left(M_{i}^{2}\right)} .
$$

Alternatively, we could have used Eq. (20) instead of Eq. (51). Using also Eq. (17) and taking real and imaginary parts, we have

$$
\begin{gathered}
m_{i}=\operatorname{Re} \sqrt{f_{i}\left(M_{i}^{2}\right)}=m_{i}^{0}-\operatorname{Re} \delta M_{i}, \\
-\frac{\Gamma_{i}}{2}=\operatorname{Im} \sqrt{f_{i}\left(M_{i}^{2}\right)}=-\operatorname{Im} \delta M_{i}
\end{gathered}
$$

where we have taken into account that the bare masses $m_{i}^{0}$ are real. By the same token, the imaginary part of $\delta M_{i}$ is UV finite, as is evident from Eq. (57).

\section{WFR bifurcation}

Let us assume temporarily that all the Majorana fermions are stable, with $\Gamma_{i}=0$ in Eq. (17). In the complex $p^{2}$ plane, their mass shells $p^{2}=m_{i}^{2}$ are then all located on the real axis below the thresholds of $\left[A_{ \pm}\left(p^{2}\right)\right]_{i j}$ and $\left[B_{ \pm}\left(p^{2}\right)\right]_{i j}$, where the absorptive parts of the latter vanish. Then, up to a sign flip in the $i \epsilon$ prescription, which is irrelevant at this stage, 
the bare propagator matrix satisfies the pseudo-Hermiticity condition $\gamma^{0}[P(\not p)]^{\dagger} \gamma^{0}=P(\not p)$ [10] 5 which implies via Eq. (8) that $\gamma^{0}[\Sigma(\not p)]^{\dagger} \gamma^{0}=\Sigma(\not p)$ [30]. In turn, this implies via Eqs. (9), (10), (13), (19), (49), (52), and (53) that

$$
\begin{aligned}
& A_{ \pm}^{\dagger}\left(p^{2}\right)=A_{\mp}\left(p^{2}\right), \quad B_{ \pm}^{\dagger}\left(p^{2}\right)=B_{ \pm}\left(p^{2}\right), \quad S_{ \pm}^{\dagger}\left(p^{2}\right)=S_{ \pm}\left(p^{2}\right), \quad T_{ \pm}^{\dagger}\left(p^{2}\right)=T_{\mp}\left(p^{2}\right), \\
& C_{ \pm}^{\dagger}\left(p^{2}\right)=D_{ \pm}\left(p^{2}\right), \quad E_{ \pm}^{\dagger}\left(p^{2}\right)=F_{ \pm}\left(p^{2}\right), \quad\left(M^{ \pm}\right)^{\dagger}=\bar{M}^{ \pm}, \\
& {\left[s_{i}\left(p^{2}\right)\right]^{*}=s_{i}\left(p^{2}\right), \quad\left[t_{i}^{ \pm}\left(p^{2}\right)\right]^{*}=t_{i}^{\mp}\left(p^{2}\right), \quad\left[f_{i}\left(p^{2}\right)\right]^{*}=f_{i}\left(p^{2}\right),}
\end{aligned}
$$

where we have used $\operatorname{adj}\left(A^{\dagger}\right)=(\operatorname{adj} A)^{\dagger}$, which follows from footnote 4, in the seventh equality. Combining Eq. (58) with Eq. (22), we find

$$
\begin{aligned}
& A_{ \pm}^{*}\left(p^{2}\right)=A_{\mp}\left(p^{2}\right), \quad B_{ \pm}^{*}\left(p^{2}\right)=B_{\mp}\left(p^{2}\right), \quad S_{ \pm}^{*}\left(p^{2}\right)=S_{\mp}\left(p^{2}\right), \quad T_{ \pm}^{*}\left(p^{2}\right)=T_{\mp}\left(p^{2}\right), \\
& C_{ \pm}^{*}\left(p^{2}\right)=C_{\mp}\left(p^{2}\right), \quad D_{ \pm}^{*}\left(p^{2}\right)=D_{\mp}\left(p^{2}\right), \quad E_{ \pm}^{*}\left(p^{2}\right)=E_{\mp}\left(p^{2}\right), \quad F_{ \pm}^{*}\left(p^{2}\right)=F_{\mp}\left(p^{2}\right), \\
& \left(M^{ \pm}\right)^{*}=M^{\mp}, \quad\left(\bar{M}^{ \pm}\right)^{*}=\bar{M}^{\mp} \text {. }
\end{aligned}
$$

The first two equalities in Eq. (59) are in agreement with Eq. (4.6) of Ref. [24]. Using the last two equalities in Eq. (58) and the one before the last in Eq. (59), we obtain from Eq. (54) that $Z_{ \pm}^{\dagger 1 / 2}=Z_{\mp}^{T^{1 / 2}}$ [24], which implies that Eqs. (25) and (27) are related as

$$
\bar{Z}^{1 / 2}=\gamma^{0} Z^{\dagger 1 / 2} \gamma^{0}
$$

From Eqs. (27) and (60), it follows that $\gamma^{0} C Z^{* 1 / 2} C^{\dagger} \gamma^{0}=Z^{1 / 2}$, so that $\Psi(x)=C\left\{[\Psi(x)]^{\dagger} \gamma^{0}\right\}^{T}$.

We now return to the general case of unstable Majorana fermions, with $\Gamma_{i}>0$ in Eq. (17). In general, we then have $\gamma^{0}[\Sigma(\not p)]^{\dagger} \gamma^{0} \neq \Sigma(\not p)$, so that Eqs. (158) and (59) no longer hold true, which enforces the departure from Eq. (60). Similar observations were made for unstable Dirac fermions at the one-loop order in Ref. [30] and to all orders in Refs. [22 23], where the notion WFR bifurcation was coined.

\section{Two-loop results}

To explore the anatomy of the all-order expressions for the renormalization constants $\delta M_{i}$ and $\left(Z_{ \pm}^{1 / 2}\right)_{i j}$ given in closed form in Eqs. (55) and (54), respectively, it is useful to perform a perturbative expansion in the generic coupling constant $\alpha$. In Ref. [24], $\delta M_{i}$ and $\left(Z_{ \pm}^{1 / 2}\right)_{i j}$ were expressed in terms of the self-energy functions $\left[A_{ \pm}\left(p^{2}\right)\right]_{i j}$ and $\left[B_{ \pm}\left(p^{2}\right)\right]_{i j}$ at the one-loop order $\mathcal{O}(\alpha)$ in a general renormalizable quantum field theory involving a mixed system of stable Majorana fermions. In the following, we assume $\left[A_{ \pm}\left(p^{2}\right)\right]_{i j}$ and $\left[B_{ \pm}\left(p^{2}\right)\right]_{i j}$ to be known through the two-loop order $\mathcal{O}\left(\alpha^{2}\right)$ and allow for the Majorana fermions to be unstable. Our goal is to express $\delta M_{i}$ and $\left(Z_{ \pm}^{1 / 2}\right)_{i j}$ in terms of $\left[A_{ \pm}\left(p^{2}\right)\right]_{i j}$

\footnotetext{
${ }^{5}$ On the right-hand side of this equation, we omitted the additional term $i \int d^{4} x e^{i p \cdot x}$ $\times\left\langle 0\left|\left[\Psi^{0}(x),\left[\Psi^{0}(0)\right]^{T} C^{*}\right]\right| 0\right\rangle$. In the noninteracting theory, its matrix elements in generation space, $\delta_{i j}\left(\frac{1}{\not p-m_{i}^{0}-i \epsilon}-\frac{1}{\not p-m_{i}^{0}+i \epsilon}\right)$, just flip the sign of the $i \epsilon$ term in $[P(\not p)]_{i j}=\frac{\delta_{i j}}{\not p-m_{i}^{0}+i \epsilon}$.
} 
and $\left[B_{ \pm}\left(p^{2}\right)\right]_{i j}$ through $\mathcal{O}\left(\alpha^{2}\right)$. In this way, we shall recover the well-known $\mathcal{O}(\alpha)$ results [24] and present the $\mathcal{O}\left(\alpha^{2}\right)$ ones for the first time.

Expanding Eqs. (53) and (46) and the factor $M_{j i}^{ \pm} / M_{i i}^{ \pm}$for $j \neq i$ in Eq. (47) through $\mathcal{O}\left(\alpha^{2}\right)$, we find

$$
\begin{aligned}
f_{i}\left(p^{2}\right)= & \frac{\left[T_{+}\left(p^{2}\right)\right]_{i i}\left[T_{-}\left(p^{2}\right)\right]_{i i}}{\left[S\left(p^{2}\right)\right]_{i i}^{2}}+m_{i}^{0}\left[\tau_{i}^{+}\left(p^{2}\right)+\tau_{i}^{-}\left(p^{2}\right)-2 m_{i}^{0} \sigma_{i}\left(p^{2}\right)\right]+\mathcal{O}\left(\alpha^{3}\right), \\
\frac{1}{\left(Z_{ \pm}^{1 / 2}\right)_{i i}^{2}}= & \frac{1-f_{i}^{\prime}\left(M_{i}^{2}\right)}{M_{i}}\left\{\left[T_{ \pm}\left(M_{i}^{2}\right)\right]_{i i}+\tau_{i}^{ \pm}\left(M_{i}^{2}\right)\right\}+\mathcal{O}\left(\alpha^{3}\right), \\
\frac{M_{j i}^{ \pm}}{M_{i i}^{ \pm}}= & \frac{1}{M_{i}^{2}-M_{j}^{2}}\left\{\left[F_{ \pm}\left(M_{i}^{2}\right)\right]_{j i}\left\{1+\frac{\left[F_{ \pm}\left(M_{i}^{2}\right)\right]_{j j}-M_{j}^{2}}{M_{i}^{2}-M_{j}^{2}}\right\}\right. \\
& \left.+\sum_{i \neq k \neq j} \frac{\left[F_{ \pm}\left(M_{i}^{2}\right)\right]_{j k}\left[F_{ \pm}\left(M_{i}^{2}\right)\right]_{k i}}{M_{i}^{2}-M_{k}^{2}}\right\}+\mathcal{O}\left(\alpha^{3}\right) \quad(j \neq i),
\end{aligned}
$$

respectively, where we have exploited the third equality in Eq. (22) to introduce $\left[S\left(p^{2}\right)\right]_{i i}=$ $\left[S_{ \pm}\left(p^{2}\right)\right]_{i i}$ and

$$
\begin{aligned}
\tau_{i}^{ \pm}\left(p^{2}\right)= & \sum_{j \neq i} \frac{\left[F_{ \pm}\left(M_{i}^{2}\right)\right]_{j i}}{M_{i}^{2}-M_{j}^{2}}\left\{m_{j}^{0} \frac{\left[F_{ \pm}\left(M_{i}^{2}\right)\right]_{j i}}{M_{i}^{2}-M_{j}^{2}}+2\left[A_{ \pm}\left(p^{2}\right)\right]_{j i}\right\} \\
\sigma_{i}\left(p^{2}\right)= & \sum_{j \neq i} \frac{1}{M_{i}^{2}-M_{j}^{2}}\left\{\frac{\left[F_{+}\left(M_{i}^{2}\right)\right]_{j i}\left[F_{-}\left(M_{i}^{2}\right)\right]_{j i}}{M_{i}^{2}-M_{j}^{2}}-\left[F_{+}\left(M_{i}^{2}\right)\right]_{j i}\left[B_{-}\left(p^{2}\right)\right]_{j i}\right. \\
& \left.-\left[F_{-}\left(M_{i}^{2}\right)\right]_{j i}\left[B_{+}\left(p^{2}\right)\right]_{j i}\right\}
\end{aligned}
$$

At this point, a few comments are in order. For $j \neq i,\left(Z_{ \pm}^{1 / 2}\right)_{j i}$ may be evaluated by substituting Eqs. (62) and (63) into Eq. (47). The first term on the right-hand side of Eq. (61) is the contribution that survives if the intergeneration mixing is turned off. For the sake of a compact notation, it is written in a factorized form, which is to be expanded through $\mathcal{O}\left(\alpha^{2}\right)$ to become

$$
\begin{aligned}
\frac{\left[T_{+}\left(p^{2}\right)\right]_{i i}\left[T_{-}\left(p^{2}\right)\right]_{i i}}{\left[S\left(p^{2}\right)\right]_{i i}^{2}}= & m_{i}^{0}\left\{m_{i}^{0}+\left[A_{+}\left(p^{2}\right)\right]_{i i}+\left[A_{-}\left(p^{2}\right)\right]_{i i}\right\}\left\{1+2\left[B\left(p^{2}\right)\right]_{i i}\right\} \\
& +\left[A_{+}\left(p^{2}\right)\right]_{i i}\left[A_{-}\left(p^{2}\right)\right]_{i i}+3\left(m_{i}^{0}\right)^{2}\left[B\left(p^{2}\right)\right]_{i i}^{2}+\mathcal{O}\left(\alpha^{3}\right),
\end{aligned}
$$

where we have used the second equality in Eq. (22) to define $\left[B\left(p^{2}\right)\right]_{i i}=\left[B_{ \pm}\left(p^{2}\right)\right]_{i i}$. In Eq. (65), it is understood that, in products of two loop functions, each factor is to be evaluated at $\mathcal{O}(\alpha)$, while loop functions that do not appear in such products are to be evaluated through $\mathcal{O}\left(\alpha^{2}\right)$. This also applies to Eqs. (61)-(64).

To express the mass counterterms $\delta M_{i}$ in Eq. (55) and the WFR matrix elements $\left(Z_{ \pm}^{1 / 2}\right)_{j i}$ in Eq. (54) in terms of renormalized parameters, we may proceed as follows. We 
first evaluate the right-hand side of Eq. (55) through $\mathcal{O}\left(\alpha^{2}\right)$ using Eq. (61) in combination with Eqs. (64) and (65). Since $\delta M_{i}$ starts at $\mathcal{O}(\alpha)$, it is sufficient to eliminate $m_{i}^{0}$ on the right-hand side of Eq. (55) using Eq. (23) with $\delta M_{i}$ evaluated to $\mathcal{O}(\alpha)$. The latter may be read off from Eqs. (61) and (65) and reads

$$
\delta M_{i}=-\frac{I_{i}\left(M_{i}^{2}\right)}{2 M_{i}}+\mathcal{O}\left(\alpha^{2}\right),
$$

with

$$
I_{i}\left(p^{2}\right)=M_{i}\left\{\left[A_{+}\left(p^{2}\right)\right]_{i i}+\left[A_{-}\left(p^{2}\right)\right]_{i i}\right\}+2 M_{i}^{2}\left[B\left(p^{2}\right)\right]_{i i},
$$

where we have replaced $m_{i}^{0}$ by $M_{i}$, with no effect to the order considered. By the same token, appearances of $m_{i}^{0}$ in the $\mathcal{O}\left(\alpha^{2}\right)$ term on the right-hand side of Eq. (55) may be replaced by $M_{i}$. Explicit and implicit appearances of $m_{j}^{0}$ with $j \neq i$ at $\mathcal{O}(\alpha)$ and $\mathcal{O}\left(\alpha^{2}\right)$ may be eliminated in the same way as those of $m_{i}^{0}$. The implicit dependence on $m_{j}^{0}$ of an $\mathcal{O}(\alpha)$ quantity, $f\left(m_{j}^{0}\right)$ say, is conveniently eliminated by Taylor expansion as $f\left(m_{j}^{0}\right)=f\left(M_{j}\right)+\delta M_{j} \partial f\left(M_{j}\right) / \partial M_{j}+\mathcal{O}\left(\alpha^{3}\right)$, where $\delta M_{j}$ is calculated to $\mathcal{O}(\alpha)$ from Eq. (66). In general, the resulting $\mathcal{O}\left(\alpha^{2}\right)$ expression for $\delta M_{i}$ still implicitly depends on other bare parameters, such as boson masses, coupling constants, and mixing-matrix elements, which also require renormalization. After this, $m_{i}^{0}$ is expressed via Eq. (23) through $\mathcal{O}\left(\alpha^{2}\right)$ entirely in terms of renormalized parameters and may thus be eliminated from Eqs. (62) and (63). Appearances of $m_{j}^{0}$ with $j \neq i$ and other bare parameters are eliminated from these equations as explained above for Eq. (55).

For $p^{2}=M_{i}^{2}$, there are some cancellations in the function $f_{i}\left(p^{2}\right)$ given by Eqs. (61), (64), and (65) through $\mathcal{O}\left(\alpha^{2}\right)$, yielding

$$
\begin{aligned}
f_{i}\left(M_{i}^{2}\right)= & \frac{\left[T_{+}\left(M_{i}^{2}\right)\right]_{i i}\left[T_{-}\left(M_{i}^{2}\right)\right]_{i i}}{\left[S\left(M_{i}^{2}\right)\right]_{i i}^{2}}+M_{i} \sum_{j \neq i}\left\{\frac{\left[F_{+}\left(M_{i}^{2}\right)\right]_{j i}}{M_{i}^{2}-M_{j}^{2}}\left\{\left[A_{+}\left(M_{i}^{2}\right)\right]_{j i}+M_{i}\left[B_{-}\left(M_{i}^{2}\right)\right]_{j i}\right\}\right. \\
& +(+\leftrightarrow-)\}+\mathcal{O}\left(\alpha^{3}\right) .
\end{aligned}
$$

The quantity $f_{i}^{\prime}\left(M_{i}^{2}\right)$ appearing in Eq. (62) is required through $\mathcal{O}\left(\alpha^{2}\right)$. Through this order, it may be conveniently evaluated as

$$
\begin{aligned}
f_{i}^{\prime}\left(M_{i}^{2}\right)= & M_{i}^{2}\left\{\frac{\left[A_{+}^{\prime}\left(M_{i}^{2}\right)\right]_{i i}}{\left[T_{+}\left(M_{i}^{2}\right)\right]_{i i}}+\frac{\left[A_{-}^{\prime}\left(M_{i}^{2}\right)\right]_{i i}}{\left[T_{-}\left(M_{i}^{2}\right)\right]_{i i}}+2 \frac{\left[B^{\prime}\left(M_{i}^{2}\right)\right]_{i i}}{\left[S\left(M_{i}^{2}\right)\right]_{i i}}\right\}+2 M_{i} \sum_{j \neq i}\left\{\frac{\left[F_{+}\left(M_{i}^{2}\right)\right]_{j i}}{M_{i}^{2}-M_{j}^{2}}\right. \\
& \left.\times\left\{\left[A_{+}^{\prime}\left(M_{i}^{2}\right)\right]_{j i}+M_{i}\left[B_{-}^{\prime}\left(M_{i}^{2}\right)\right]_{j i}\right\}+(+\leftrightarrow-)\right\}+\mathcal{O}\left(\alpha^{3}\right),
\end{aligned}
$$

where we have used Eq. (51) through $\mathcal{O}(\alpha)$ to eliminate the combination on the left-hand side of Eq. (65). The functions $\left[F_{ \pm}\left(p^{2}\right)\right]_{j i}$ with $j \neq i$ are required through $\mathcal{O}\left(\alpha^{2}\right)$ at their first appearance in Eq. (63) and through $\mathcal{O}(\alpha)$ elsewhere in Eqs. (63), (64), (68), and 
(69). Through $\mathcal{O}\left(\alpha^{2}\right)$, we have

$$
\begin{aligned}
{\left[F_{ \pm}\left(p^{2}\right)\right]_{j i}=} & M_{j}\left\{\left[I_{N}+B_{\mp}\left(p^{2}\right)\right] A_{ \pm}\left(p^{2}\right)\right\}_{j i}+\left[A_{\mp}\left(p^{2}\right)+A_{\mp}\left(p^{2}\right) B_{\mp}\left(p^{2}\right)+B_{ \pm}\left(p^{2}\right) A_{\mp}\left(p^{2}\right)\right]_{j i} M_{i} \\
& +\left\{B_{ \pm}\left(p^{2}\right)\left[I_{N}+B_{ \pm}\left(p^{2}\right)\right]\right\}_{j i} M_{i}^{2}+M_{j}\left\{B_{\mp}\left(p^{2}\right)\left[I_{N}+B_{\mp}\left(p^{2}\right)\right]\right\}_{j i} M_{i} \\
& +\left[A_{\mp}\left(p^{2}\right) A_{ \pm}\left(p^{2}\right)\right]_{j i}+\sum_{k}\left[B_{ \pm}\left(p^{2}\right)\right]_{j k} M_{k}\left\{\left[A_{ \pm}\left(p^{2}\right)\right]_{k i}+\left[B_{\mp}\left(p^{2}\right)\right]_{k i} M_{i}\right\} \\
& +\delta M_{j}\left\{\left[A_{ \pm}\left(p^{2}\right)\right]_{j i}+\left[B_{\mp}\left(p^{2}\right)\right]_{j i} M_{i}\right\}+\left\{\left[A_{\mp}\left(p^{2}\right)\right]_{j i}+M_{j}\left[B_{\mp}\left(p^{2}\right)\right]_{j i}\right. \\
& \left.+2\left[B_{ \pm}\left(p^{2}\right)\right]_{j i} M_{i}\right\} \delta M_{i}+\mathcal{O}\left(\alpha^{3}\right) .
\end{aligned}
$$

Finally, the combination $\left[F_{ \pm}\left(M_{i}^{2}\right)\right]_{j j}-M_{j}^{2}$ appearing in Eq. (63) is required through $\mathcal{O}(\alpha)$, where it may be rewritten in terms of the function $I_{i}\left(p^{2}\right)$ in Eq. (67) as

$$
\left[F_{ \pm}\left(M_{i}^{2}\right)\right]_{j j}-M_{j}^{2}=I_{j}\left(M_{i}^{2}\right)-I_{j}\left(M_{j}^{2}\right)+\mathcal{O}\left(\alpha^{2}\right) .
$$

In the case of stable Majorana fermions, in which Eqs. (58) and (59) apply, the $\mathcal{O}(\alpha)$ terms of Eqs. (46), (47), and (55) evaluated using Eqs. (61)-(63) and (65) agree with Eqs. (4.11), (4.10), and (4.12) of Ref. [24], respectively.

\section{Conclusions}

We renormalized the propagator matrix of a mixed system of unstable Majorana fermions in a general parity-nonconserving quantum field theory adopting the pole scheme, in which the pole masses serve as the renormalized masses. The squares of the pole masses are the complex poles of the propagator matrix. The inverse propagator matrix is built up by the one-particle-irreducible Feynman diagrams pertaining to the transitions of fermion $j$ to fermion $i$ order by order in perturbation theory. In gauge theories, the pole masses are expected to be gauge independent. This was proven for the SM [18] using Nielsen identities [19]. In spontaneously broken gauge theories, one needs to include the tadpoles to ensure the gauge independence of the mass counterterms. This then carries over to the pole masses because the bare masses are gauge independent as a matter of principle.

The WFR matrices were determined by requiring that each diagonal element of the renormalized propagator matrix resonates with unit residue if the respective fermion is on its mass shell. This renormalization condition is singled out by the LSZ reduction formalism [21] because it avoids finite renormalizations that are otherwise required. In this sense, it may be considered scheme independent. Furthermore, it uniquely fixes the WFR matrices. This is in contrast to the Dirac case, where some residual freedom exists, which may be exhausted by imposing an additional WFR condition [22 2324]. Specifically, this residual freedom affects the pairs of WFR matrix elements that appear as factors in the off-diagonal entries of the renormalized propagator matrix. As for Dirac fermions [22 2330], we encountered WFR bifurcation in the case of instability, i.e., the WFR matrices of the in and out states are no longer related by Hermitian conjugation, so that Eq. (60) is violated. However, they are still related by Eq. (27), which is a consequence of the Majorana condition in Eq. ([6) and is absent in the Dirac case. 
The dressed propagator matrix and the renormalization constants are expressed in terms of the unrenormalized self-energies of the $j \rightarrow i$ transitions, which have scalar, pseudoscalar, vector, and axial vector parts. Owing to the Majorana condition in Eq. (66), the latter are subject to the symmetry relations given by the first two equalities in Eq. (22). We presented closed analytic results, which are valid to all orders because we refrained from explicit perturbative expansions. Specifically, the renormalized dressed propagator matrix is given by Eq. (32), the pole mass counterterms by Eq. (55), and the WFR matrices by Eq. (54). In these formulas, the renormalized masses $M_{i}$ enter as arguments $p^{2}=M_{i}^{2}$ of the various self-energy functions, and it is understood that the latter are evaluated from the bare Lagrangian of the considered quantum field theory, so that the masses, couplings, and mixing angles on which they depend are all bare parameters to start with.

Apart from being interesting in their own right, the results presented here have a number of important phenomenological applications. In the following, we mention but three of them. First, in the perturbative treatment of a specific particle scattering or decay process involving stable or unstable Majorana fermions, our formulas for $\delta M_{i}$ and $\left(Z_{ \pm}^{1 / 2}\right)_{i j}$ may be used after expansion through the considered order and truncation of terms beyond that order. If the unstable Majorana fermion $i$ occurs on an internal line, then $\delta M_{i}$ enters. If it occurs on an external line, then $\left(Z_{ \pm}^{1 / 2}\right)_{i j}$ enters. Strictly speaking, unstable particles are not entitled to appear in asymptotic states of scattering amplitudes in quantum field theory. However, in numerous applications of significant phenomenological interest, the rigorous compliance with this tenet would immediately entail a proliferation of external legs and bring the evaluation of radiative corrections to a grinding halt, the more so as almost all the known elementary particles are unstable. For the reader's convenience, we presented explicit two-loop expressions for the renormalization constants, in Eqs. (61) -(71), which may be employed in phenomenological applications involving Majorana fermions as they stand. In the one-loop case of stable Majorana fermions, Eqs. (4.10)-(4.12) in Ref. [24] are reproduced by Eqs. (47), (46)), and (55), respectively, evaluated at $\mathcal{O}(\alpha)$ using Eqs. (61) - (63) and (65) .

Second, the total decay widths $\Gamma_{i}$ may be perturbatively evaluated through any order from the unrenormalized self-energy functions $\left[A_{ \pm}\left(p^{2}\right)\right]_{i j}$ and $\left[B_{ \pm}\left(p^{2}\right)\right]_{i j}$ by solving Eq. (57) iteratively.

Third, our result for the mass counterterms $\delta M_{i}$ may be used to switch from the pole scheme adopted here to any other scheme of mass renormalization, as long as the method of regularization is maintained. In fact, since the bare masses $m_{i}^{0}$ are independent of the choice of renormalization scheme, the equivalent of Eq. (23) in some other scheme is

$$
m_{i}^{0}=\tilde{M}_{i}+\delta \tilde{M}_{i},
$$

where $\delta \tilde{M}_{i}$ has the same UV singularities as $\delta M_{i}$, but differs in the finite terms. In gauge theories, preferable renormalization schemes are those in which $\delta \tilde{M}_{i}$ is arranged to be gauge independent, so that $\tilde{M}_{i}$ enjoys the same desirably property. Equating Eqs. (23) and (72), we thus obtain a UV-finite relationship between the renormalized masses of 
both schemes,

$$
M_{i}=\tilde{M}_{i}+\delta \tilde{M}_{i}-\delta M_{i} .
$$

A quantity evaluated to a given order of perturbation theory in the pole scheme may then be translated to the other mass renormalization scheme by substituting Eq. (73), expanding in the coupling constant, and discarding terms beyond the considered order.

In the context of perturbative calculations in quantum chromodynamics, the modified minimal-subtraction $(\overline{\mathrm{MS}})$ scheme [33] of dimensional regularization 34 is frequently employed in the literature. A natural extension of the $\overline{\mathrm{MS}}$ definition of mass to the EW sector of the SM may be obtained from Eq. (56) by writing

$$
\begin{aligned}
m_{i}^{0} & =m_{i}+\operatorname{Re} \delta M_{i} \\
& =\bar{m}_{i}+\delta \bar{m}_{i},
\end{aligned}
$$

where

$$
\delta \bar{m}_{i}=\left(\operatorname{Re} \delta M_{i}\right)_{\mathrm{UV}}
$$

collects just the poles in $\varepsilon=2-d / 2$, with $d$ being the dimensionality of space-time, and the familiar terms involving $\gamma_{E}-\ln (4 \pi)$, with $\gamma_{E}$ being Euler's constant, that appear in $\operatorname{Re} \delta M_{i}$ order by order. The latter may be absorbed by an appropriate redefinition of the renormalization scale $\mu$, namely, $\mu=\mu^{\prime} \exp \left(\gamma_{E} / 2\right) /(2 \sqrt{\pi})$ [35]. From Eqs. (74) and (75), it hence follows that

$$
\begin{aligned}
m_{i} & =\bar{m}_{i}+\left(\operatorname{Re} \delta M_{i}\right)_{\mathrm{UV}}-\operatorname{Re} \delta M_{i} \\
& =\bar{m}_{i}-\left(\operatorname{Re} \delta M_{i}\right)_{\overline{\mathrm{MS}}},
\end{aligned}
$$

where $\left(\operatorname{Re} \delta M_{i}\right)_{\overline{\mathrm{MS}}}$ is the UV-finite remainder of $\operatorname{Re} \delta M_{i}$ after $\overline{\mathrm{MS}}$ subtraction of the poles in $\varepsilon$ at renormalization scale $\mu$. As mentioned above, it is necessary to include the tadpole contributions in $\left(\operatorname{Re} \delta M_{i}\right)_{\overline{\mathrm{MS}}}$ in order for $\bar{m}_{i}$ to be gauge independent [36]. Otherwise, the functional dependencies of radiatively corrected transition matrix elements on such renormalized masses acquire artificial gauge dependence, and the choices of gauge must always be specified along with the values of such renormalized masses extracted from experimental data. The necessity to include the tadpole contributions in order to render the mass counterterms gauge independent was also noticed within the on-shell renormalization of the SM at one loop [9]1137]. In that case, however, the omission of the tadpole contributions would be inconsequential in practice, since the functional dependencies of radiatively corrected transition matrix elements on the renormalized masses could be preserved at the expense of allowing for the bare masses to become gauge dependent. Unfortunately, such an escape is unavailable in the case of Eq. (76) , which directly relates the mass definitions in two different renormalization schemes [36].

\section{Acknowledegments}

We thank Alberto Sirlin for numerous valuable discussions and Concha Gonzalez-Garcia for an illuminating communication regarding Ref. [6]. This research was supported in 
part by the German Research Foundation through the Collaborative Research Center No. SFB 676 Particles, Strings and the Early Universe-The Structure of Matter and Space Time.

\section{References}

[1] G. Aad et al. (ATLAS Collaboration), Phys. Lett. B 716, 1 (2012) arXiv:1207.7214 [hep-ex]]; S. Chatrchyan et al. (CMS Collaboration), ibid. 716, 30 (2012) arXiv:1207.7235 [hep-ex]].

[2] F. Bezrukov, M. Yu. Kalmykov, B. A. Kniehl, and M. Shaposhnikov, J. High Energy Phys. 10 (2012) 140 [arXiv:1205.2893 [hep-ph]].

[3] G. Degrassi, S. Di Vita, J. Elias-Miró, J. R. Espinosa, G. F. Giudice, G. Isidori, and A. Strumia, J. High Energy Phys. 08 (2012) 098 [arXiv:1205.6497 [hep-ph]]; D. Buttazzo, G. Degrassi, P. P. Giardino, G. F. Giudice, F. Sala, A. Salvio, and A. Strumia, ibid. 12 (2013) 089 [arXiv:1307.3536 [hep-ph]].

[4] N. V. Krasnikov, Yad. Fiz. 28, 549 (1978) [Sov. J. Nucl. Phys. 28, 279 (1978)]; H. D. Politzer and S. Wolfram, Phys. Lett. B 82, 242 (1979); 83, 421(E) (1979); P. Q. Hung, Phys. Rev. Lett. 42, 873 (1979).

[5] J. Beringer et al. (Particle Data Group), Phys. Rev. D 86, 010001 (2012).

[6] M. C. Gonzalez-Garcia, PoS(ICHEP2012)005.

[7] P. Minkowski, Phys. Lett. B 67, 421 (1977); R. N. Mohapatra and G. Senjanović, Phys. Rev. Lett. 44, 912 (1980); T. Yanagida, Prog. Theor. Phys. 64, 1103 (1980); J. Schechter and J. W. F. Valle, Phys. Rev. D 22, 2227 (1980).

[8] G. 't Hooft, Nucl. Phys. B33, 173 (1971); J. C. Taylor, ibid. B33, 436 (1971); G. 't Hooft, ibid. B35, 167 (1971); B. W. Lee, Phys. Rev. D 5, 823 (1972); G. 't Hooft and M. Veltman, Nucl. Phys. B50, 318 (1972); D. A. Ross and J. C. Taylor, ibid. B51, 125 (1973); B58, 643(E) (1973).

[9] J. Fleischer and F. Jegerlehner, Phys. Rev. D 23, 2001 (1981).

[10] K.-i. Aoki, Z. Hioki, R. Kawabe, M. Konuma, and T. Muta, Prog. Theor. Phys. Suppl. 73, 1 (1982).

[11] M. Böhm, H. Spiesberger, and W. Hollik, Fortsch. Phys. 34, 687 (1986).

[12] W. F. L. Hollik, Fortsch. Phys. 38, 165 (1990).

[13] A. Sirlin, Phys. Rev. D 22, 971 (1980). 
[14] P. A. Grassi, Nucl. Phys. B462, 524 (1996) hep-th/9505101); E. Kraus and K. Sibold, Nucl. Phys. B (Proc. Suppl.) 51C, 81 (1996) |hep-th/9608143]; E. Kraus, Annals Phys. (N.Y.) 262, 155 (1998) [hep-th/9709154]; P. A. Grassi, T. Hurth, and M. Steinhauser, ibid. 288, 197 (2001) [hep-ph/9907426].

[15] N. Cabibbo, Phys. Rev. Lett. 10, 531 (1963); M. Kobayashi and T. Maskawa, Prog. Theor. Phys. 49, 652 (1973).

[16] B. Pontecorvo, Zh. Eksp. Teor. Fiz. 33, 549 (1957) [Sov. Phys. JETP 6, 429 (1958)]; Zh. Eksp. Teor. Fiz. 34, 247 (1958) [Sov. Phys. JETP 7, 172 (1958)]; Z. Maki, M. Nakagawa, and S. Sakata, Prog. Theor. Phys. 28, 870 (1962); B. Pontecorvo, Zh. Eksp. Teor. Fiz. 53, 1717 (1967) [Sov. Phys. JETP 26, 984 (1968)].

[17] J. F. Donoghue, Phys. Rev. D 19, 2772 (1979).

[18] P. Gambino and P. A. Grassi, Phys. Rev. D 62, 076002 (2000) [hep-ph/9907254].

[19] N. K. Nielsen, Nucl. Phys. B101, 173 (1975).

[20] B. A. Kniehl and A. Sirlin, Phys. Rev. D 85, 036007 (2012) arXiv:1201.4333 [hep$\mathrm{ph}]$.

[21] H. Lehmann, K. Symanzik, and W. Zimmermann, Nuovo Cimento 1, 205 (1955); 6, 319 (1957).

[22] B. A. Kniehl, Phys. Rev. Lett. 112, 071603 (2014) [arXiv:1308.3140 [hep-ph]].

[23] B. A. Kniehl, Phys. Rev. D 89, 096005 (2014).

[24] B. A. Kniehl and A. Pilaftsis, Nucl. Phys. B474, 286 (1996) hep-ph/9601390.

[25] K.-P. O. Diener and B. A. Kniehl, Nucl. Phys. B617, 291 (2001) hep-ph/0109110.

[26] A. A. Almasy, B. A. Kniehl, and A. Sirlin, Nucl. Phys. B818, 115 (2009) arXiv:0902.3793 [hep-ph]].

[27] B. A. Kniehl and A. Sirlin, Phys. Rev. Lett. 97, 221801 (2006) hep-ph/0608306; Phys. Rev. D 74, 116003 (2006) [hep-th/0612033].

[28] B. A. Kniehl and A. Sirlin, Phys. Lett. B 673, 208 (2009) arXiv:0901.0114 [hep-ph]]; AIP Conf. Proc. 1182, 327 (2009) [arXiv:0906.2670 [hep-ph]].

[29] F. J. Dyson, Phys. Rev. 75, 1736 (1949).

[30] D. Espriu, J. Manzano, and P. Talavera, Phys. Rev. D 66, 076002 (2002) hep-ph/0204085. 
[31] M. C. Smith and S. S. Willenbrock, Phys. Rev. Lett. 79, 3825 (1997) hep-ph/9612329]; M. Passera and A. Sirlin, Phys. Rev. D 58, 113010 (1998) hep-ph/9804309.

[32] B. A. Kniehl and A. Sirlin, Phys. Rev. D 77, 116012 (2008) [arXiv:0801.0669 [hepth]].

[33] W. A. Bardeen, A. J. Buras, D. W. Duke, and T. Muta, Phys. Rev. D 18, 3998 (1978).

[34] C. G. Bollini and J. J. Giambiagi, Nuovo Cimento B 12, 20 (1972); G. 't Hooft and M. Veltman, Nucl. Phys. B44, 189 (1972); J. F. Ashmore, Lett. Nuovo Cimento 4, 289 (1972); Commun. Math. Phys. 29, 177 (1973).

[35] B. A. Kniehl, Phys. Rev. D 50, 3314 (1994) hep-ph/9405299.

[36] R. Hempfling and B. A. Kniehl, Phys. Rev. D 51, 1386 (1995) hep-ph/9408313; F. Jegerlehner and M. Yu. Kalmykov, Nucl. Phys. B676, 365 (2004) [hep-ph/0308216]; M. Faisst, J. H. Kühn, and O. Veretin, Phys. Lett. B 589, 35 (2004) (hep-ph/0403026]; B. A. Kniehl, J. H. Piclum, and M. Steinhauser, Nucl. Phys. B695, 199 (2004) hep-ph/0406254; F. Jegerlehner, M. Yu. Kalmykov, and B. A. Kniehl, Phys. Lett. B 722, 123 (2013) arXiv:1212.4319 [hep-ph]]; PoS(DIS 2013)19 [arXiv:1307.4226 [hep-ph]]; B. A. Kniehl and O. L. Veretin, Nucl. Phys. B885, 459 (2014) [arXiv:1401.1844 [hep-ph]].

[37] A. Sirlin and R. Zucchini, Nucl. Phys. B266, 389 (1986); G. Degrassi and A. Sirlin, ibid. B383, 73 (1992). 